\title{
Crystal structure of $\mathrm{LaTiO}_{3.41}$ under pressure
}

\author{
I. Loa ${ }^{\circledast}$ K. Syassen, and X. Wang \\ Max-Planck-Institut für Festkörperforschung, Heisenbergstraße 1, D-70569 Stuttgart, Germany \\ F. Lichtenberg \\ Institut für Physik, EKM, Experimentalphysik VI, Universität Augsburg, \\ Universitätsstraße 1, D-86135 Augsburg, Germany \\ M. Hanfland \\ European Synchrotron Radiation Facility, BP 220, F-38043 Grenoble, France \\ C. A. Kuntscher \\ Universität Stuttgart, 1. Physikalisches Institut, Pfaffenwaldring 57, D-70550 Stuttgart, Germany
}

(Dated: November 11, 2018)

\begin{abstract}
The crystal structure of the layered, perovskite-related $\mathrm{LaTiO}_{3.41}\left(\mathrm{La}_{5} \mathrm{Ti}_{5} \mathrm{O}_{17+\delta}\right)$ has been studied by synchrotron powder x-ray diffraction under hydrostatic pressure up to $27 \mathrm{GPa}(T=295 \mathrm{~K})$. The ambient-pressure phase was found to remain stable up to $18 \mathrm{GPa}$. A sluggish, but reversible phase transition occurs in the range 18-24 GPa. The structural changes of the low-pressure phase are characterized by a pronounced anisotropy in the axis compressibilities, which are at a ratio of approximately $1: 2: 3$ for the $a, b$, and $c$ axes. Possible effects of pressure on the electronic properties of $\mathrm{LaTiO}_{3.41}$ are discussed.
\end{abstract}

PACS numbers: $\quad 61.50 . \mathrm{Ks}, 61.10 . \mathrm{Nz}, 72.80 . \mathrm{Ga}, 71.38 .+\mathrm{i}, 71.30 .+\mathrm{h}$

\section{INTRODUCTION}

$\mathrm{LaTiO}_{3.41}$ belongs to a series of perovskite-related layered compounds of the composition $A_{n} B_{n} \mathrm{O}_{3 n+2}$ $\mathrm{LaTiO}_{3.41}$ is a $n=5$ member of this class, and thus it may alternatively be denoted as $\mathrm{La}_{5} \mathrm{Ti}_{5} \mathrm{O}_{17+\delta}$. Its monoclinic crystal structure is illustrated in Fig. 10 The samples studied here and in previous work $\mathrm{k}^{1.2 .5}$ have a slight oxygen excess of $0.3 \%$ and are therefore denoted as $\mathrm{LaTiO}_{3.41}$ instead of $\mathrm{LaTiO}_{3.40}$.

As a consequence of the oxygen-rich composition of $\mathrm{LaTiO}_{3.41}$ (compared to $\mathrm{LaTiO}_{3}$ ), its Ti-3d derived electronic bands are only partially occupied $\left(\mathrm{Ti} 3 d^{0.18}\right)$. Electrical transport measurements ${ }^{2.5}$ on $\mathrm{LaTiO}_{3.41}$ revealed strongly anisotropic properties and a quasi-1D metallic behavior. The DC resistivity along the $b$ direction and perpendicular to the $a b$ planes showed semiconducting behavior in the temperature range of 4-290 K. Along the $a$ direction a metal-like temperature dependence of the conductivity was reported for the temperature range of $60-200 \mathrm{~K}$. Above $200 \mathrm{~K}$ a slight decrease in resistivity with increasing temperature was observed, indicating conduction due to polaron hopping ${ }^{2.5}$ Below $60 \mathrm{~K}$ the resistivity increased steeply, corresponding to an electronic activation energy of $\sim 8 \mathrm{meV}$. The apparent opening of an electronic band gap has been discussed in terms of a possible Peierls distortion of this quasi-1D system ${ }^{2}$

A study of the mid- and far-infrared optical properties of $\mathrm{LaTiO}_{3.41}$ has recently corroborated its quasi-1D metallic character $\frac{5}{5}$ Furthermore, signatures of polaronic charge carriers were found in the form of a mid-infrared band with pronounced temperature-dependent changes. The polaron scenario is in overall agreement with the ex-
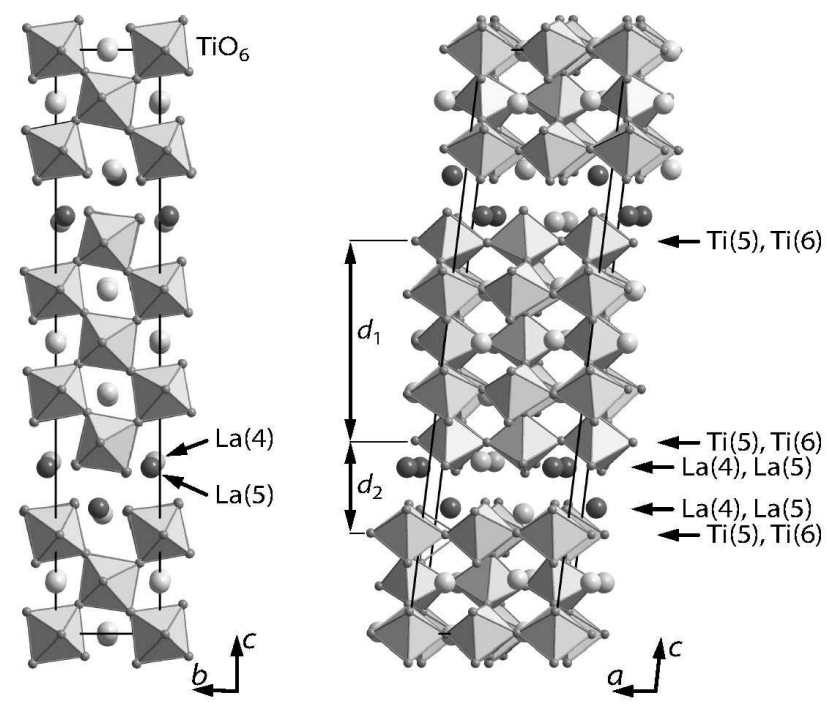

FIG. 1: The monoclinic crystal structure $\frac{1}{\underline{1}}$ of $\mathrm{LaTiO}_{3.41}$ at ambient conditions (space group $P 2_{1} / c, Z=4$ ) can be represented by slabs of corner-sharing $\mathrm{TiO}_{6}$ octahedra, separated by layers of additional oxygen atoms. Within the slabs of thickness $d_{1}$, the octahedra are connected and tilted cooperatively in the same fashion as in the more familiar $\mathrm{GeFeO}_{3}$-type distorted perovskites such as $\mathrm{LaTiO}_{3}$. La ions are located in the voids between the octahedra.

perimental results obtained so far. The exact nature of the polaronic carriers, however, still needs to be clarified.

The application of high hydrostatic pressure provides a tool to tune the structural and the electronic properties of $\mathrm{LaTiO}_{3.41}$. On the one hand, this may offer a possibility to drive the system more into a quasi-1D metallic regime, 
i.e. over a wider range of temperatures. On the other hand, and more importantly, it allows to investigate the nature of the charge carriers, in particular their polaronic character, as was detailed by Goddat et al $l^{6}$

Here we explore the structural changes of $\mathrm{LaTiO}_{3.41}$ under hydrostatic pressures up to $27 \mathrm{GPa}$. The crucial question of the structural stability of the ambientpressure phase is addressed. The pressure-induced structural changes of the low-pressure phase are analyzed and the anticipated changes in the electronic system discussed. We aim at providing a basis for the interpretation of future investigations of the physical properties of $\mathrm{LaTiO}_{3.41}$ at high pressures.

\section{EXPERIMENTAL DETAILS}

The structural properties of $\mathrm{LaTiO}_{3.41}$ under pressure were studied up to $27 \mathrm{GPa}$ and ambient temperature by monochromatic $(\lambda=0.4176 \AA)$ x-ray powder diffraction at the European Synchrotron Radiation Facility (ESRF Grenoble, beamline ID9A). A crystal was ground finely and some powder placed into a diamond anvil cell (DAC) for pressure generation. Nitrogen served as a pressure transmitting medium to provide nearly hydrostatic conditions. Diffraction patterns were recorded with an image plate detector and then integrated ${ }^{7}$ to yield intensity vs. $2 \theta$ diagrams. The DAC was rotated by $\pm 3^{\circ}$ during the exposure to improve the powder averaging. Pressures were determined with the ruby luminescence method $\stackrel{8}{*}$ The diffraction diagrams were analyzed with the Rietveld method, i.e. whole pattern fitting, using the GSAS software ${ }^{9.10}$ The synthesis of the $\mathrm{LaTiO}_{3.41}$ material has been described elsewhere ${ }^{2}$

\section{RESULTS AND DISCUSSION}

Figure 2(a) shows x-ray diffraction diagrams of $\mathrm{LaTiO}_{3.41}$ for increasing pressures up to $24 \mathrm{GPa}$. At pressures above $2 \mathrm{GPa}$ additional reflections (mostly weak) due to various phases of solid nitrogen were observed. Up to $18 \mathrm{GPa}$, there are no discontinuous changes in the diffraction patterns of $\mathrm{LaTiO}_{3.41}$.

At pressures above $18 \mathrm{GPa}$ a structural phase transition is evidenced by the appearance of additional reflections. The transition is completed at $24 \mathrm{GPa}$. There is a continuous evolution of the low-angle (002) reflection of the low-pressure phase towards a corresponding peak in the high-pressure diagrams [inset of Fig. [2(a)]. It suggests that the long $c$ axis $\left(c_{0}=31.5 \AA\right)$ is preserved across the phase transition. As a result, there are a large number of overlapping reflections, so that a unique determination of the high-pressure unit cell has not been possible. The structural phase transition is reversible.

A rather small increase in the widths of the reflections $(\sim 6 \%)$ before and after the $0 \rightarrow 27 \rightarrow 0$ GPa pressure

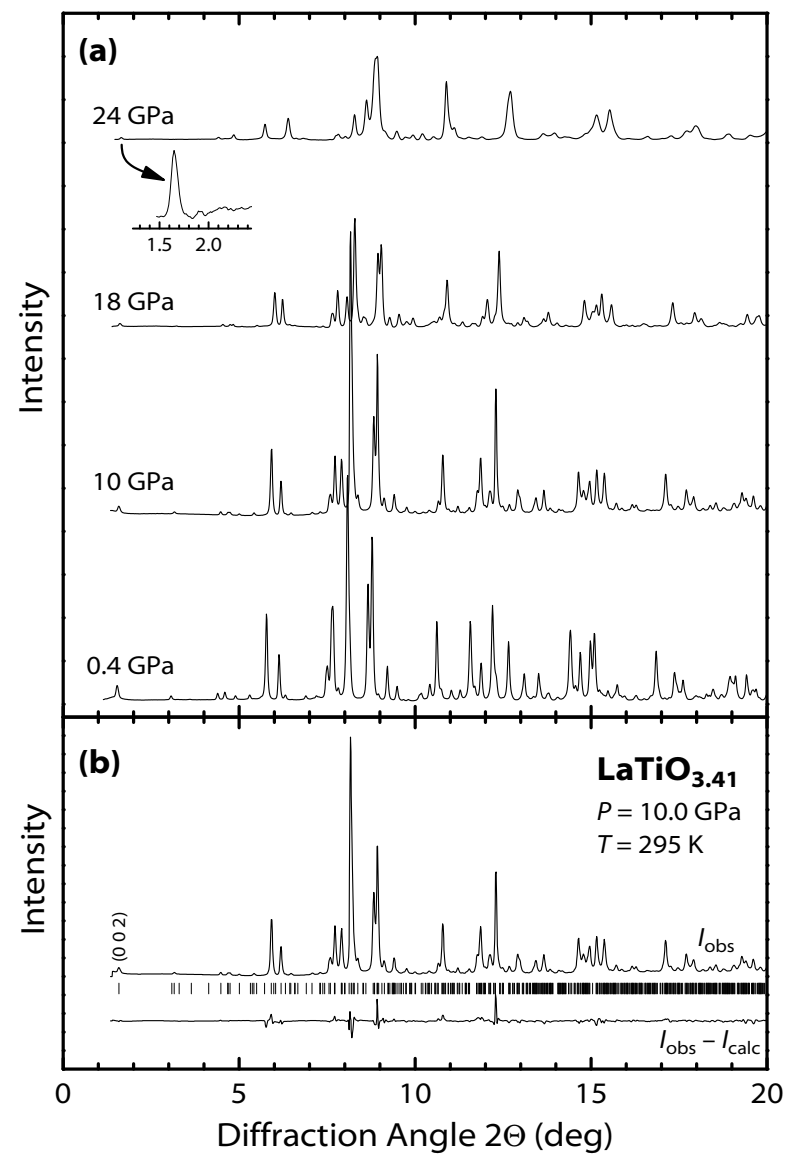

FIG. 2: (a) Diffraction diagrams of $\mathrm{LaTiO}_{3.41}$ at high pressures $(\lambda=0.4176 \AA)$. The diffractogram of the high-pressure phase at $24 \mathrm{GPa}$ shows a line at $1.65^{\circ}$ (inset), corresponding to the (002) reflection of the ambient-pressure phase. (b) X-ray powder diffraction diagram $\left(I_{\text {obs }}\right)$ of $\mathrm{LaTiO}_{3.41}$ at $10.0 \mathrm{GPa}$ and difference curve $\left(I_{\text {obs }}-I_{\text {calc }}\right)$ for a partial Rietveld refinement (La and Ti positions only). Markers show the calculated peak positions.

cycle indicates the creation of only a small amount of defects during the phase transformations. It suggests that the phase transition may represent a distortion of the low-pressure crystal structure rather than a reconstructive transition.

The lattice parameters of the low-pressure phase as a function of pressure were determined from Rietveld-type fits of the diffraction diagrams. This approach was chosen over a direct determination on the basis of the peak positions because of the massive overlap of reflections [Fig. 2(b)]. The lattice parameters were determined up to $18 \mathrm{GPa}$ as shown in Fig. 31(a). The compression under hydrostatic pressure is rather anisotropic. The relative compressibilities of the $a, b$, and $c$ directions are at a ratio of approximately $1: 2: 3$.

The inset of Fig. 3(b) shows that the monoclinic angle increases slightly (from 97.17 to 97.43 ) with increasing pressure to $18 \mathrm{GPa}$. It can be determined in two ways: either directly from the refinements (i. e., from the 


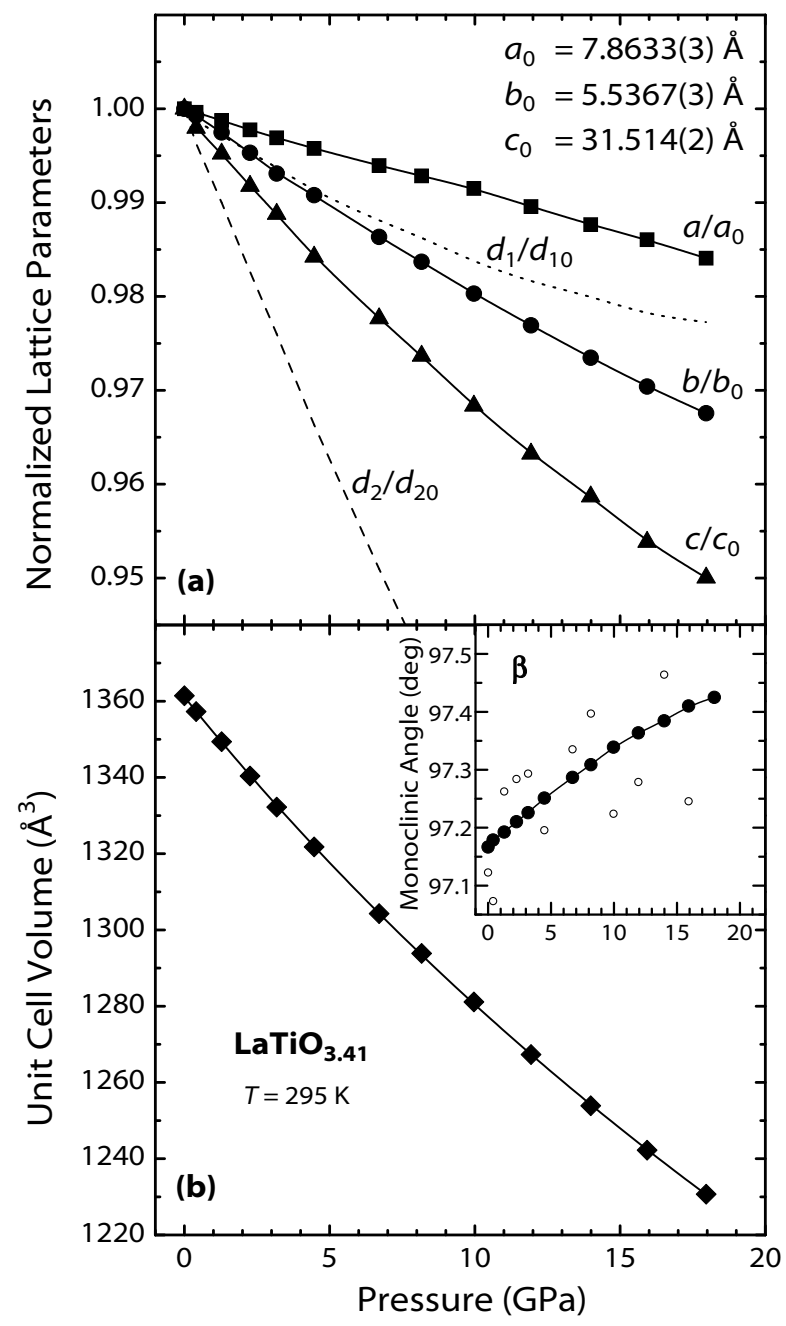

FIG. 3: Structural parameters of $\mathrm{LaTiO}_{3.41}$ as a function of pressure. (a) Lattice parameters $a, b, c$ as well as estimates of the slab thickness $d_{1}$ and interlayer separation $d_{2}$, normalized to their respective zero-pressure values. The zeropressure values of $d_{1}$ and $d_{2}$ amount to $d_{10} \approx 11.14 \AA$ and $d_{20} \approx 4.50 \AA$, respectively. See text for details. (b) The experimental pressure-volume data can be represented by a Murnaghan equation of state (solid line). The inset shows the variation of the monoclinic angle with pressure. Solid symbols represent angles determined from the lattice parameters $a$ and $c$; open symbols refer to the results of the refinements.

peak positions) or from the ratio of the lattice parameters $a$ and $c$. The latter is possible because there are two identical building blocks per unit cell that are shifted by $a / 4$ along the $a$ direction with respect to each other [Fig. 1. As a consequence, the monoclinic angle $\beta$ is fully determined by the lattice parameters $a$ and $c$ through $\beta=\arccos (a / 2 c)$. The values of $\beta$ calculated in this way have much smaller uncertainties than those derived directly from the Rietveld refinements, see Fig. 31(b).

From the lattice parameters we calculate the unit cell volume as a function of pressure as shown in Fig. 3(b). The experimental data are well represented by the Mur- naghan relation ${ }^{11} V(P)=V_{0}\left[\left(B^{\prime} / B_{0}\right) P+1\right]^{-1 / B^{\prime}}$ with the bulk modulus $B_{0}=142.2(11) \mathrm{GPa}$ and its derivative $B^{\prime}=4.3(2)$ at zero pressure. Here the ambient-pressure unit cell volume was kept fixed at the experimental value of $V_{0}=1361.5(2) \AA^{3}$. The ambient-pressure unit cell volume determined here is $\sim 0.4 \%$ larger than reported previously $\underline{\underline{1}}$

In order to explore the origin of the anisotropic compressibility, it is worthwhile to estimate, as a function of pressure, the thicknesses $d_{1}$ and $d_{2}$ of the $\mathrm{LaTiO}_{3}-$ type slabs and the separating layers, respectively [Fig. 1]. A measure of these quantities is provided by the $z$ coordinates of the $\operatorname{Ti}(5)$ and $\operatorname{Ti}(6)$ ions that are adjacent to the oxygen-rich layers. Therefore, an attempt was made to determine the atomic positions of the heavier atoms La and Ti by means of Rietveld refinements. Here one should bear in mind that the unit cell of $\mathrm{LaTiO}_{3.41}$ contains 20 atoms each of $\mathrm{La}$ and $\mathrm{Ti}$ with $15 \mathrm{La}$ atomic coordinates and $12 \mathrm{Ti}$ coordinates to be determined. This is at the borderline of what can be extracted from highpressure x-ray powder diffraction data.

Figure 4 displays the variation with pressure of the $\operatorname{Ti}(5)$ and $\operatorname{Ti}(6) z$-coordinates. It is evident that the scatter in the values determined directly from the refinements (smaller open symbols) originates primarily from a correlation of larger $\operatorname{Ti}(5)$ with smaller $\operatorname{Ti}(6) z$ positions. At ambient conditions, the $\operatorname{Ti}(5)$ and $\operatorname{Ti}(6)$ ions are located at $z=0.1781 \pm 0.0006$, i.e., almost within a common plane. This is very similar to the $\mathrm{LaTiO}_{3}$ case where the corresponding $\mathrm{Ti}$ atoms are placed strictly in a single plane. Altogether, assuming equal $z$ parameters for the $\operatorname{Ti}(5)$ and $\operatorname{Ti}(6)$ atoms should provide a good approximation to the actual situation. The averaged $\mathrm{Ti}(5,6)$ $z$ coordinates show a clear variation with pressure, see Fig. 团

From the $\operatorname{Ti}(5,6) z$ coordinates we can estimate the thicknesses $d_{1}$ of the $\mathrm{LaTiO}_{3}$-type slabs and $d_{2}$ of the separating layers [cf. Fig. 1]. Figure 3(a) shows that the variation of $d_{1}$ with pressure is comparable to that of the crystallographic $a$ and $b$ directions, while the interlayer spacing $d_{2}$ is much more compressible. The large compressibility along $c$ therefore results from the highly compressible oxygen-rich layers where the rather strong $\mathrm{Ti}-\mathrm{O}$ bonds are missing. This large difference in compressibilities also explains the increase of the monoclinic angle with pressure [inset of Fig [3(b)].

As a check on the plausibility of the estimated $d_{1}, d_{2}$ values we have calculated the bulk modulus of the $\mathrm{LaTiO}_{3}$-type layers; it amounts to $B_{0} \approx 190 \mathrm{GPa}$. While there seems to be no experimental data on the bulk modulus of $\mathrm{LaTiO}_{3}$ available for comparison, our estimate is close to the bulk modulus $B_{0}=194(3)$ GPa of perovskitetype $\mathrm{YTiO}_{3} \frac{12}{12}$ Hence, the $\operatorname{Ti}(5,6)$ positions and the layer thicknesses derived therefrom can be considered sufficiently accurate to gain some insight into the structural changes within the unit cell.

Additional information on the structural changes near the layer boundaries can be obtained from the $\mathrm{La}(4)$ and 


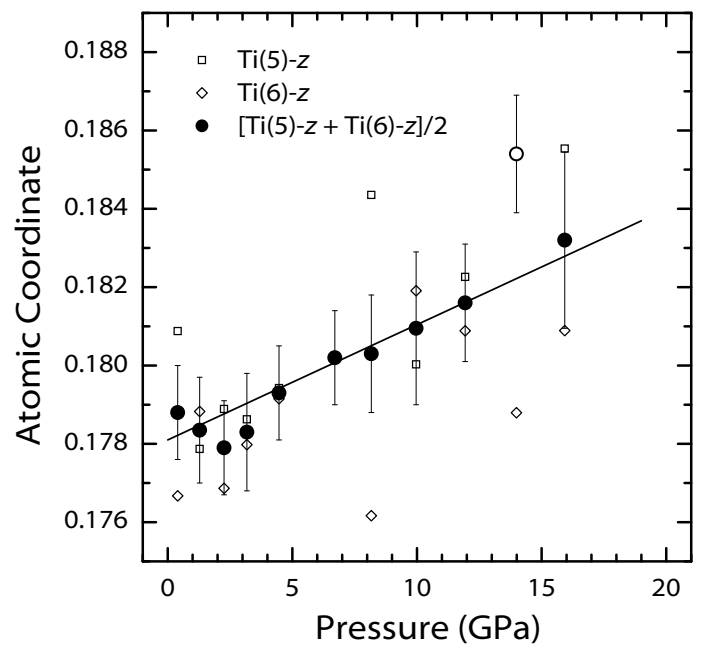

FIG. 4: Evolution of the $\operatorname{Ti}(5)$ and $\operatorname{Ti}(6) z$ coordinates as a function of pressure. Open squares and diamonds represent the $\operatorname{Ti}(5)$ and $\operatorname{Ti}(6)$ data, respectively, as determined in the Rietveld refinements. These data scatter in a correlated fashion. The circles show the averaged $z$ coordinates of $\mathrm{Ti}(5)$ and $\operatorname{Ti}(6)$. The solid line represents a linear fit to the data $[z(P) \approx 0.1781+0.00029 P / \mathrm{GPa}]$, with the outlier at $14 \mathrm{GPa}$ (open circle) not being taken into account. The error bars mark three times the uncertainties reported by the refinement program GSAS.

$\mathrm{La}(5)$ positions. At ambient conditions both of these ions are shifted from their regular positions $(z=1 / 3)$ between the $\mathrm{TiO}_{6}$ octahedra into the interlayer region [Fig. 1]. This displacement is more pronounced for $\mathrm{La}(5)$ than for $\mathrm{La}(4)$. Figure 5 illustrates that La(4) hardly moves along $c$ under pressure, while $\mathrm{La}(5)$ shifts towards the $\mathrm{LaTiO}_{3}-$ slab region. With regard to the displacement off the $a c$ plane $(y=0), \mathrm{La}(5)$ moves continuously towards this plane with increasing pressure while $\mathrm{La}(4)$ appears to be slightly more displaced at intermediate pressures. Altogether, pressure forces the $\mathrm{La}(4)$ and $\mathrm{La}(5)$ ions closer to the "ideal" positions in the ac planes, they adopt similar positions along the $c$ direction, but they remain displaced into the interlayer region.

With respect to the electronic properties of $\mathrm{LaTiO}_{3.41}$ (and any other distorted perovskite structure), the tilting of the $\mathrm{TiO}_{6}$ octahedra represents a key information. A measurement of these tiltings in the case of $\mathrm{LaTiO}_{3.41}$ would require an accurate determination of the 51 oxygen atomic coordinates which does not appear feasible on the basis of an x-ray powder diffraction experiment. It is possible, however, to arrive at a semi-quantitative estimate of the tilt angles under pressure on the basis of the lattice parameters and the slab thickness $d_{1}$. The relation between octahedral tilting and orthorhombic distortion of the unit cell is well known for the threedimensionally linked perovskites of $\mathrm{ABO}_{3}$ type $\mathbf{1 3 . 1 4 . 1 5}^{13.15}$ The same scheme can be applied to the $\mathrm{LaTiO}_{3}$-type slabs of $\mathrm{LaTiO}_{3.41}$, where the octahedra are connected and tilted in the same fashion. In the following, $d_{1} / 2$

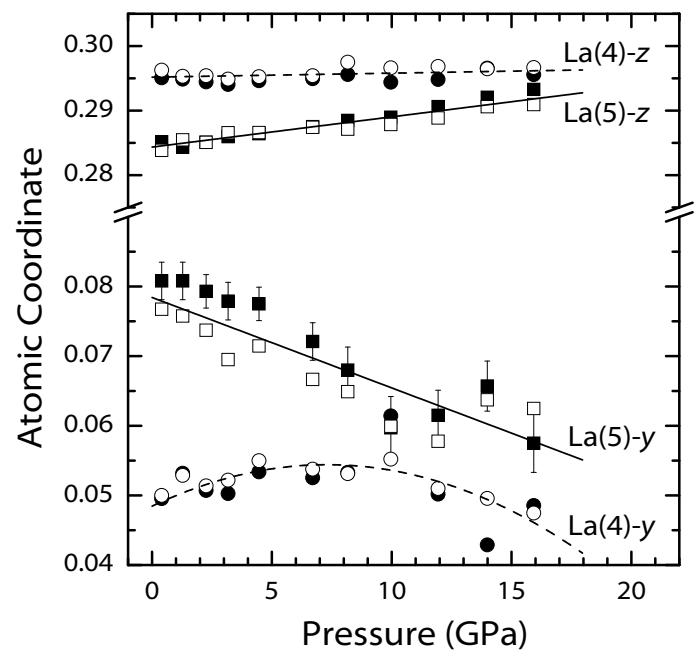

FIG. 5: Evolution of the $\mathrm{La}(4)$ and $\mathrm{La}(5)$ atomic coordinates $y$ and $z$ as a function of pressure. Closed (open) symbols refer to refinement results where preferred orientation effects were (were not) taken into account. The error bars mark three times the uncertainties of the La- $y$ coordinates reported by the refinement program GSAS. For clarity, they are shown only for one data set. In the case of the La- $z$ coordinates, the uncertainties are similar to the symbol size. Lines are guides to the eye.

takes the role of the third lattice parameter besides $a$ and $b$. On the basis of the equations given by O'Keeffe and Hyde, $\stackrel{13}{,}$ we use the relations

$$
\varphi\left(a, b, d_{1}\right)=\arccos \left(\frac{\sqrt{8} b^{2}}{a d_{1}}\right)
$$

and

$$
\varphi(a, b)=\arccos \left(6(b / a)^{2}-2\right)
$$

to determine the octahedral tilt angles shown in Fig. [6] The geometrical constraints of the cooperative octahedral tilting that are at the origin of these relations and the definition of $\varphi$ are detailed in Ref. 13. Evaluation of the tilt angle on the basis of Eq. (11) with three lattice parameters is generally less sensitive to distortions of the octahedra than the two-parameter determination. The important result is that both approaches indicate a significant increase in the average octahedral tilt angle $\varphi$ with increasing pressure. Roughly, the tilting angle doubles at $18 \mathrm{GPa}$ compared to ambient conditions.

The indication of a pressure-induced increase in octahedral tilt represents an interesting difference in comparison to other transition metal perovskites such as

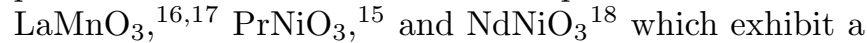
reduction in the tilt. While there are certainly no universal pressure-induced changes in the $\mathrm{GdFeO}_{3}$-type perovskite compounds, as has been pointed out before, 15 there may exist a systematic behavior across the series of $R T \mathrm{O}_{3}$ rare-earth transition-metal oxides that appears worthwhile to be explored. 


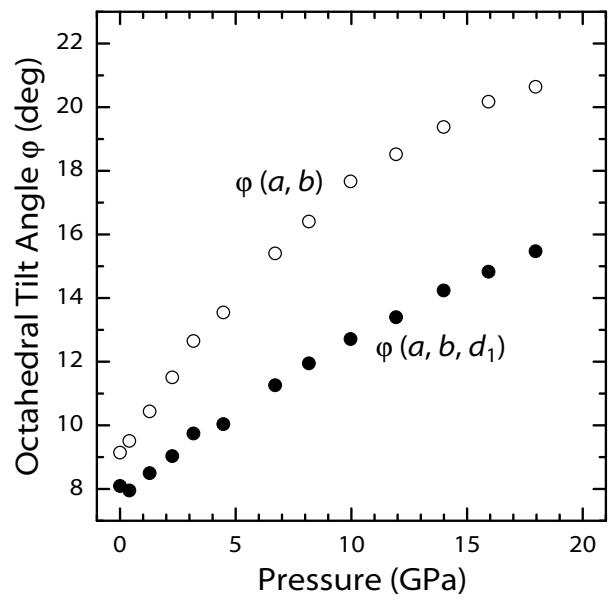

FIG. 6: Octahedral tilt angles $\varphi$ vs pressure derived from the lattice parameters $a$ and $b$ and the slab thickness $d_{1}$ according to Eqns (1) and (2).

Stability of $\mathrm{LaTiO}_{3.41}$ under pressures of up to $18 \mathrm{GPa}$ opens way to tune its electrical transport properties. On the one hand, one can vary the itinerancy of the system that is determined by the $\mathrm{Ti}-\mathrm{O}$ bond lengths and $\mathrm{Ti}-$ $\mathrm{O}-\mathrm{Ti}$ bond angles. A reduction in bond lengths results in a larger bandwidth $W$ of the electronic bands derived from the $t_{2 g}$-orbitals and an enhanced itinerancy of the system, while a decrease in the bond angles (increased octahedral tilt) has the opposite effect. Hence, we have here two competing pressure-induced changes that will affect the itinerancy of the system. These changes are most important for the quasi-1D metallic state that exists in the temperature range of $60-200 \mathrm{~K}$ at ambient pressure. On the other hand, polaron binding energies are generally expected to decrease with increasing pressure due to the stiffening of the lattice ${ }^{6}$ Therefore, highpressure optical and/or electrical transport experiments are promising approaches to test the hypothesis on polaronic conductivity in the quasi-1D $\mathrm{LaTiO}_{3.41}$.

In conclusion, we have studied the crystal structure of $\mathrm{LaTiO}_{3.41}$ by synchrotron x-ray powder diffraction up to $27 \mathrm{GPa}$. The ambient-pressure phase remains stable up to $18 \mathrm{GPa}$ (at $295 \mathrm{~K}$ ). Above $18 \mathrm{GPa}$ a sluggish phase transition occurs, which is completed at $24 \mathrm{GPa}$. The low-pressure phase is characterized by a pronounced anisotropy of the axis compressibilities at a ratio of approximately $1: 2: 3$ for the $a, b$ and $c$ axes. The anisotropy can be related to rather compressible oxygenrich layers that separate the perovskite-type slabs and to variations in the tilt angles of the $\mathrm{TiO}_{6}$ octahedra. Stability of $\mathrm{LaTiO}_{3.41}$ up to $18 \mathrm{GPa}$ opens way to tune its electronic transport properties over a relatively large range. It motivates further electrical transport and optical experiments to study the unusual transport properties of $\mathrm{LaTiO}_{3.41}$ in more detail.
* Corresponding author: E-mail I.Loa@fkf.mpg.de

1 P. Daniels, F. Lichtenberg, and S. van Smaalen, Acta Cryst. C 59, i15 (2003).

${ }^{2}$ F. Lichtenberg, A. Herrnberger, K. Wiedenmann, and J. Mannhart, Prog. Solid State Chem. 29, 1 (2001).

3 J. Galy and A. Carpy, Phil. Mag. 29, 1207 (1974).

${ }^{4}$ F. Lichtenberg, D. Widmer, J. G. Bednorz, T. Williams, and A. Reller, Z. Phys. B 82, 211 (1991).

${ }^{5}$ C. A. Kuntscher, D. van der Marel, M. Dressel, F. Lichtenberg, and J. Mannhart, Phys. Rev. B 67, 035105 (2003).

6 A. Goddat, J. Peyronneau, and J. P. Poirier, Phys. Chem. Minerals 27, 81 (1999).

7 A. Hammersley, computer program FiT2D, (ESRF, Grenoble, 1998).

${ }^{8}$ H. K. Mao, J. Xu, and P. M. Bell, J. Geophys. Res. 91, 4673 (1986).

9 A. C. Larson and R. B. von Dreele, GSAS: General Structure Analysis System. Report LAUR 86-748, Los Alamos National Laboratory, NM, USA (1986).

10 Owing to the large number of adjustable atomic coordinates $(15 \times \mathrm{La}, 12 \times \mathrm{Ti}, 51 \times \mathrm{O})$, only the positions of the heavier atoms $\mathrm{La}$ and $\mathrm{Ti}$ were refined. Usually, the analysis was performed in at least two ways, i. e. without and with preferred orientation corrections applied. The dif- ferences in the results mark the experimental uncertainty. Preferred orientation effects were modeled using spherical harmonics. A simple pseudo-Voigt line shape was sufficient to describe the reflection profiles up to the highest pressures.

11 F. D. Murnaghan, Proc. Nat. Acad. Sci. USA 30, 244 (1944).

12 X. Wang, I. Loa, K. Syassen et al. (unpublished).

13 M. O'Keeffe and B. G. Hyde, Acta Cryst. B 33, 3802 (1977).

14 A. M. Glazer, Acta Cryst. B 28, 3384 (1972).

15 M. Medarde, J. Mesot, P. Lacorre, S. Rosenkranz, P. Fischer, and K. Gobrecht, Phys. Rev. B 52, 9248 (1995).

16 I. Loa, P. Adler, A. Grzechnik, K. Syassen, U. Schwarz, M. Hanfland, G. K. Rozenberg, P. Gorodetsky, and M. P. Pasternak, Phys. Rev. Lett. 87, 125501 (2001).

17 L. Pinsard-Gaudart, J. Rodríguez-Carvajal, A. DaoudAladine, I. Goncharenko, M. Medarde, R. I. Smith, and A. Revcolevschi, Phys. Rev. B 64, 064426 (2001).

$18 \mathrm{M}$. Amboage, $\mathrm{RNiO}_{3}$ peroskites under high pressure ( $R=$ rare earth or $Y$ ), Ph.D. thesis, The University of the Basque Country, Spain (2003). 